\title{
Framework for Resilient Production Systems
}

\author{
Matthias Heinicke \\ Otto von Guericke University Magdeburg, Universitätsplatz 2, 39106 Magdeburg, Germany \\ Matthias.heinicke@ovgu.de
}

\begin{abstract}
To respond to turbulent changes in customer demand in a flexible and timely manner, an adequate configuration of production systems in terms of an increasing agility is crucial. In contrast, robust processes, which avoid or at least reduce any kind of dissipation, are increasingly in the focus of practitioners. This includes standardized material and information flows. Furthermore, the concept of resilience combines both agility and robustness and represents the ability of a system to cope with change effectively. This paper highlights the relevance of production control as regards the implementation of resilience in an organizational context. Against this backdrop, a functional map revealing the interactions of the individual tasks of production planning and control enables a systematic analysis of the causes of dissipation. Moreover, socio-technical aspects in terms of the identification of hierarchically structured roles within these activities are considered.
\end{abstract}

Keywords: resilience, functional map, production control, configuration.

\section{Introduction}

Various influencing factors work on an existing production system. Accordingly, their impact is currently represented mainly by the effort of increased agility in recent years. This concept includes increased responsiveness to changing environmental conditions (customer demand, pricing pressures, and supplier development). Consequently, an anticipation and adaptation to the turbulence of the environment is imperative. However, the ideal measures to meet these requirements in various fields are discussed in literature intensively as regards resilience (Bhamra et al., 2011). Resilience serves as means in order to cope with turbulence (Wieland and Wallenburg, 2013; Ivanov and Sokolov, 2013). However, its impact and effects require thorough empirical evidence.

In productions systems it is aimed at avoiding or at least reduction of dissipation. In this context, dissipation describes all actions or events in terms of perturbations, which affect efficiency and lower productivity. To overcome the consequent issues resulting from turbulence, various efforts are made within the scope of production planning and control. Particularly production control aims at reliable and robust production processes ensuring a rapidly response to changes and the achievement of production targets despite turbulent environmental influences. However, an accurate 
execution of control principles often fails due to a lack of understanding of the interdependencies and effects (Schuh et al., 2013).

Therefore, this paper introduces an approach providing a framework for analysing the interactions of the individual tasks of production planning and control. Thus, transparency is increased and operational users are enabled to track the information flow in this regard. The approach additionally intends to reveal interactions between environmental conditions and organizational aspects of production systems in the context of ensuring a reliable operation of organizational processes. For this purpose, a hierarchical pattern is deduced to specify the roles and the corresponding tasks of production planning and control. The focus of the following approach is the optimization of the transformation process as regards the avoidance of operational and organizational errors.

\section{Definition of Resilient Production Processes}

\subsection{Agility and Robustness in an Organizational Context}

Robustness is the property of a system to resist change or external influences without adapting its initial stable configuration and continuously provide a desired output (Ivanov and Sokolov, 2013; Wieland and Wallenburg, 2012). Accordingly, a robust system is insensitive to disturbances of the system during its operation (Zhang and Luttervelt, 2011). In general, robustness refers to a proactive strategy, which prevents supplier-related volatility from restricting the functionality of a supply chain (Wieland and Wallenburg, 2012) and thus production processes also. This concept implies resistance to anticipated changes (Wieland and Wallenburg, 2013).

The possibilities to design a robust system configuration are limited to ongoing internal and external changes. In this context, path dependency (Codes and Hülsmann, 2013) is a crucial factor in terms of an appropriate determination of a specific agility level. In addition to the robustness requirement, agility enables a reaction to severe disturbances that cannot be endured by the robustness of a production system (Ivanov and Sokolov, 2013). Identifying the correct manner and time of response to this kind of influence is crucial for the sustainability of production. The concept of speed is inherent to agility (Wieland and Wallenburg, 2012; Bhamra et al., 2011). Agility refers to a quickly and adequate response to unexpected changes and thus is similar to flexibility (Ivanov and Sokolov, 2013). Therefore, a perception of current changes is imperative for a fast reaction to perceived issues (Wieland and Wallenburg, 2013).

\subsection{Resilience in an Organizational Context}

The concept of resilience contains ecological, social, individual/psychological and organizational/socio-technical aspects (Bhamra et al., 2011). Resilience always is the ability of a system to cope with change (Wieland and Wallenburg, 2013). In the context of production systems, the resilience domain combines two dimensions: agility, which expresses reactive strategies, and robustness including proactive strategies (Wieland and Wallenburg, 2013); thus it is similar to changeability (Wiendahl, 2002). The first one meets environmental changes with corresponding organizational action 
by re-configuring operating states of a prodution system rapidly and flexibly. The latter is based on forecasting and prevention. Thus, robust systems endure rather than respond to changes due to the preservation of a stable system configuration. As a result, resilience implies self-regulation and resistance to disturbances of a system (Bhamra et al., 2011).

In summary, operational and organizational robustness expresses the ability of a system obtaining its functionality even under fluctuating environmental conditions. With respect to the resilience definition, both aspects, robustness and agility, have to be considered in terms of a closed-loop control system as regards production systems. Minor perturbations endured by the robustness of the system differ from grave disturbances that require a rapid re-configuration of the production system based on its agility property.

\section{Resilience by the Means of Production Control}

\subsection{Influencing Production Systems by Production Control}

In addition to production planning, the main factor to influence existing production systems represents production control. Production planning produces an image of the desired target state by the generation of default values for manufacturing and assembly. Empirically, the target state will not occur due to uncertainties as well as disturbances during production. In this context, production control ensures compliance with the desired management objectives (lead time, capacity utilization, inventory, on-time delivery) by intervening in current production processes. Based on the production program, results of the respective upstream planning level within the operational volume planning, scheduling and capacity levelling represent the input data for the next item. This is called cascaded loops (Nyhuis et al., 2009). Figure 1 illustrates a cascaded loop in production, which represents a hierarchical pattern of individual functions. For this purpose, the respective input and output variables of the individual viewing areas are represented and analyzed in their dependency and interaction (cf. Section 4.2).

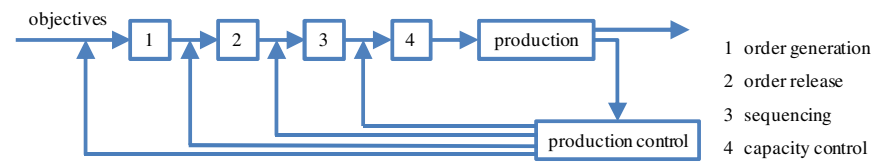

Fig. 1. Cascaded closed-loop production control

Unforeseen dissipation in a production system necessarily effect that its targets cannot be met. Consequently, in these cases an intervention at an operational level is required to ensure compliance with deadlines and other objectives. It is assumed that certain interactions between the various functional areas exist within production systems (Zülch, 1990). In this context, the kind of order release, the sequence of production orders and the batch size (order generation) are crucial parameters (Nyhuis et al., 2006). The combination of these parameters enables specific control methods 
(e.g. kanban, load-dependent order release, etc.). According to the presented task groups, following strategies in the production control can be distinguished:

- $\quad$ strategies for determining batch size,

- $\quad$ strategies for resource allocation (selection and scheduling),

- $\quad$ strategies for sequencing and

- $\quad$ strategies for order release.

A reasonable combination of these strategies is crucial. In this context, for employees both informal and formal degrees of freedom apply in exercising their functions.

\subsection{Deficits in Production Control}

In contrast to the deterministic effects assumed by existing approaches in terms of production control configuration, the influence of socio-technical aspects on the performance of control principles is nearly neglected (Schuh et al., 2013). In this context, various problems in the accurate implementation of control principles result from the diversity of choice, a lack of communication and a deficient comprehension of the effects of decisions. Therefore, degrees of freedom as a function of the applied control method, the specific task within the production control as well as in consideration of the respective groups of people (production planners, foremen, and workers) necessitate a clear definition in terms of the hierarchical structure of control processes. It is assumed that within production systems people adapt processes and its control. Regardless of the existing configuration of a production system in terms of robustness and agility, all the persons involved affect the level of its resilience.

Production planners primarily control the flow of information as regards production program. Task of foremen is the organization of the production processes. Workers will perform the actual manufacturing techniques and processes. In this context, it is assumed that the formal rules of the production control in terms of their effectiveness are limited in multistage production systems. This entails other organizational rules for trapping occurring negative effects that cannot be confined by common control principles. Therefore, identifying an appropriate operating point regarding the balancing between the degree of freedom of the employees and the deterministic execution of control principles is imperative depending on factors of influence and disturbances.

\section{Approach for Resilient Production Systems}

\subsection{Closed Loop of the Tasks of Production Control}

Production control serves to determine or rather anticipate derivations and to make the necessary adjustments accordingly. A proper balance between stability in terms of robust processes and dynamic adaption based on the agility of the system can be realized by appropriate principles of production control (Schuh et al., 2013). Thus, production control and adequate decisions of people implement the concept of resilience 
within production systems. For an expedient adaption by decision, it is essential to identify the cause-effect relationships for the individual operational targets in advance. Thus, this enables a positioning of the production in the tension of operational objectives.

A robust order processing in compliance with the logistic objectives therefore requires the mapping and analysis of all relevant processes and their interdependencies (Schuh and Stich 2, 2012). The efficiency of production is determined by a variety of factors. In this context, order generation and order release represent possibilities to influence the manufacturing system directly. In contrast, organization and availability of resources are two aspects that individual persons hardly can work on. Those can be characterized as random and thus are not directly affected by the decisions of the production control. The production resources include equipment, personnel and material. A lack of availability leads to a decline of the efficiency and the intrinsic flexibility of production. A reduction of availability even by disturbing environmental factors is possible. The organization provides a restrictive framework for the objectives, which exists before the implementation of production control. Predominantly, the workplace layout, the legal guidelines, the process principle of the production (operational organization) as well as the flow of information are determined as the most important factors that affect the attainable targets in advance.

\subsection{Functional Map of Production Control Tasks}

During a production process, variations and disturbances that complicate the control of production typically occur. Plan deviations appearing at different points of the production processes can be of various kinds and entail different consequences. In this context, robustness is measured in form of deviations as regards the four logistic objectives mentioned above. In order to protect against deviations, a high transparency in the planning and control processes is necessary. The functional map (Figure 2) represents such a tool depicting the individual tasks of production planning and control dependent on the hierarchy levels. The concatenating representation of the entire planning and controlling activities includes the appropriate dissemination of information. This tool depicts a self-contained and interactive structure. Thus, effects of a re-configuration of the flow of information and the scope for decision-making are assessable. Thereby, the functional map allows for the determination of the causes of problems as regards occurring deviations or faults. In addition, this facilitates coping with such disturbances by adopting effective measures quickly. The feedback of the results obtained in terms of data is very important to initiate improvements in a timely manner. Moreover, it is crucial that an adequate communication between the hierarchical levels proceeds (Schuh and Stich 1, 2012). As a result, the functional map systematically reveals both potentials and capabilities regarding the ideal interaction of the tasks of production planning and control. Thus, it supports the operational process of decision making. 


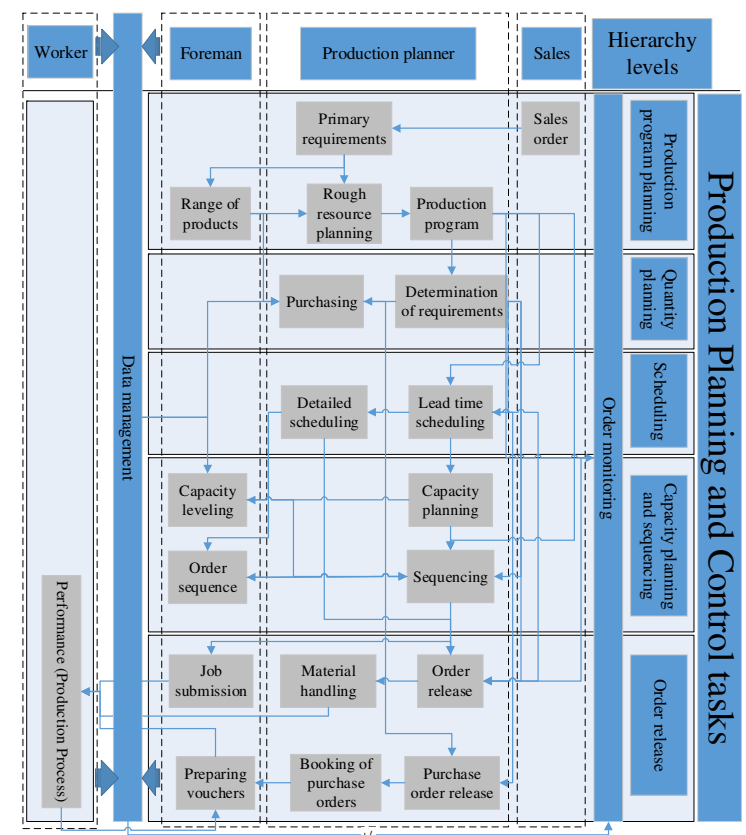

Fig. 2. Functional map of the basic tasks of production control

\subsection{Implementation of Robust Processes}

A robust configuration of manufacturing systems needs to meet several requirements. In this context, it is aimed at an accurate and controllable execution of tasks as regards fault prevention by anticipation (occurrence of errors) as well as reducing the effects of disturbances due to preparedness (coping with errors). Moreover, robustness implies various operational objectives. Hereby, the focus is on a comparably qualitative and quantitative yield of repetitive production processes. This requires a precise definition of all operations and a reliable understanding of one's role within the hierarchical structure of the production system. In addition, operational key figures should be insensitive to disturbances of a production based on robust processes. In this context, robustness of production systems is assessable by the degree of change in the achievement of objectives in terms of production-related indicators as a function of environmental factors (Jodlbauer, 2008). This implies that the production program is fulfilled in spite of unforeseen variations reliably.

Furthermore, a clear understanding of the course of processes within in the production system is imperative. Apart from the production control activities, all inputoutput-relations of the execution of orders have to be considered. For that purpose, the functional map reduces the complexity of the entire production planning and control by a classification of individual tasks with respect to hierarchy levels. Thus, the effects of respective measures can be assessed easily. To realize insensitiveness to disturbances, potential fault causes has to be determined first. Subsequently, an identification of potential effects of disturbances allows a definition of the scope and manner of 
measures for each hierarchy level. At the backdrop of the implementation of robust processes, this contribution offers an approach managing all issues related to the identification and elimination of the effects of disturbances. In consideration of each operational objective, the following steps have to be passed:

- Identification of influences on the production system,

- Classification in disturbance variable and control variable, and

- $\quad$ Prioritization as regards controllability as well as relevance.

A cause and effect diagram supports the identification of influences. Hence, all potential influences are collected and well-structured. Due to the interdependencies of management goals, the analysis in terms of influence on production systems has to be conducted for each objective separately. After collecting all influences, identified issues should undergo a classification. In consideration of the amount of influences, it is differentiated between control and disturbance variables. The crucial difference is the potential exertion of influence. Disturbance variables represent external or environmental aspects. In contrast, some issues can be controlled within a production system. Lastly, every detected influence is assessed as regards controllability and relevance. The first dimension represents the differentiation between control and disturbance variable. The relevance aspect is an indicator for the effects of corresponding influences. It is assumed that the total time an influence factor impedes production processes represents an adequate key figure for relevance. Prioritizing influences enables operational users to take the ideal measures. With respect to different hierarchy levels, the individual scope of action is crucial in this regard.

\subsection{Beyond Robust Production Systems}

In consideration of organizationally robust processes, a limitation of the scope of action and decision of the individual process owners is required regarding the sequence and priority of individual orders. This consequently affects the departmental job control in terms of self-organization and self-optimization (Hartmann, 1998). Thus, if the robustness is no longer sufficient, the agility properties are claimed. Moreover, an establishment of a defined corridor of flexibility is necessary (Terkaj et al., 2009). In this context, various aspects of changeability come into consideration (Wiendahl, 2002). To meet the requirements of a turbulent environment, an examination of the effects of structural and organizational aspects of agility is imperative. Furthermore, the focus is on recognizing the need for changes of production systems in terms of structure (elements and resources) or organization (organizational structure and operational organization).

\section{Conclusion and Future Work}

In summary, this paper introduces an approach that combines socio-technical aspects and deterministic interactions of the tasks of production control. Due to the fact that several operators are unable to cope with complex control principles, their involvement is imperative in order to estimate the effects of the freedom of choice as regards 
the individual tasks of production control. The functional map enables an analysis of key levers influencing production systems. Based on the hierarchical pattern possibilities of intervention are drawn systematically, so that operational users will be in a position to act independently within coordinated control loops. Production control executed by operational users enables an adaptive balancing between robustness and agility and thus represents the implementation of the concept of resilience within production systems. However, the more intricate the production system and its organization are, the more difficult the application of this approach seems to be. In this regard, additional research is necessary.

\section{References}

Bhamra, R., Dani, S., Burnard, K.: Resilience: The concept, a literature review and future directions. International Journal of Production Research 49(18), 5375-5393 (2011)

Ivanov, D., Sokolov, B.: Control and system-theoretic identification of the supply chain dynamics domain for planning, analysis and adaption of performance under uncertainty. European Journal of Operational Research 224(2), 313-323 (2013)

Wieland, A., Wallenburg, C.: Dealing with supply chain risks. International Journal of Physical Distribution \& Logistics Management 42(10), 887-905 (2012)

Wieland, A., Wallenburg, C.M.: The influence of relational competencies on supply chain resilience. International Journal of Physical Distribution \& Logistics Management 43(4), 300-320 (2013)

Schuh, G., Potente, T., Thomas, C.: Design of Production Control's Behavior. In: Forty Sixth CIRP Conference on Manufacturing Systems 2013, pp. 145-150 (2013)

Zhang, W.J., van Luttervelt, C.A.: Toward a resilient manufacturing system. CIRP Annals Manufacturing Technology 60(1), 469-472 (2011)

Cordes, P., Hülsmann, M.: Dynamic Capabilities in Manufacturing Processes: A Knowledge-based Approach for the Development of Manufacturing Flexibilities. In: Windt, K. (ed.) Robust Manufacturing Control, pp. 519-534. Springer, Berlin (2013)

Nyhuis, P., Hartmann, W., Kennemann, M., Münzberg, B.: Produktionsregelung mit logistischen Kennlinien. wt Werkstattstechnik 99(4), 239-243 (2009)

Nyhuis, P., Begemann, C., Berkholz, D., Hasenfuß, K.: Konfiguration der Fertigungssteuerung. wt Werkstattstechnik 96(4), 195-199 (2006)

Zülch, G.: Systematisierung von Strategien der Fertigungssteuerung. In: Zahn, E., Bullinger, H. (eds.) Organisationsstrategie und Produktion, pp. 151-178. Gfmt, München (1990)

Schuh, G., Stich, V.: Produktionsplanung und -steuerung 2: Evolution der PPS. Springer, Berlin (2012)

Schuh, G., Stich, V.: Produktionsplanung und -steuerung 1: Grundlagen der PPS. Springer, Berlin (2012)

Jodlbauer, H.: Produktionsoptimierung. Springer, Berlin (2008)

Hartmann, M.: Merkmale zur Wandlungsfähigkeit von Produktionssystemen bei turbulenten Aufgaben. GESIS, Barleben (1998)

Terkaj, W., Tolio, T., Valente, A.: Focused Flexibility in Production Systems. In: Elmaraghy, H.A. (ed.) Changeable and Reconfigurable Manufacturing Systems, pp. 47-66. Springer (2009)

Wiendahl, H.: Wandlungsfähigkeit: Schlüsselbegriff der zukunftsfähigen Fabrik. Werkstattstechnik 2002 92(4), 122-127 (2002) 